\title{
On Quadratic Lagrangians in General Relativity
}

\author{
E. Pechlaner and R. SexL \\ Institute for Theoretical Physics, University of Vienna, Vienna, Austria
}

Received October 8, 1965

Abstract. Theories of gravitation similar to General Relativity but with an additional $R^{2}$ term in the Lagrangian are explored. The Schwarzschild metric is not the exterior solution that can be continued to the interior of the body to give a positive definite mass distribution. The experimental consequences of $R^{2}$ terms are investigated. Furthermore, it is shown that a theory with an $R^{2}$ term only possesses an interesting singular dependence on the coupling constant.

\section{Introduction}

One of the requirements Einstein made in deriving the equations of General Relativity was that the field equations should contain only second derivatives and these only linearly. Postulating this and a coupling of the energy-momentum tensor to the metric of a Riemannian space time, Ensstein was led almost uniquely to the famous field equations of General Relativity:

$$
R_{\mu \nu}-\frac{1}{2} g_{\mu \nu} \cdot R=-\varkappa \cdot T_{\mu \nu}
$$

derivable from an action integral

$$
W=\int d^{4} x(\sqrt{-g} \cdot R+2 \varkappa \cdot \Lambda(x))
$$

where $\Lambda(x)$ is the action integral of the matter fields.

From Quantum Electrodynamics we know that the vacuum polarization introduces nonlinear terms into the originally linear equations of this theory. There, the classical Lagrangian

$$
L=\frac{1}{2}\left(\mathbf{E}^{2}-\mathbf{H}^{2}\right)
$$

has to be modified to

$$
L=\frac{1}{2}\left(\mathbf{E}^{2}-\mathbf{H}^{2}\right)+\frac{2 \alpha^{2}}{45 m^{4}}\left[\left(\mathbf{E}^{2}-\mathbf{H}^{2}\right)^{2}+7(\mathbf{E} \cdot \mathbf{H})^{2}\right]
$$

( $\alpha$ is the fine-structure constant and $m$ the electron mass, we put $c=1$ ) to account for the effects of vacuum polarization in the first nonvanishing order in the coupling constant.

The same phenomenon is likely to occur in the quantisation of General Relativity, although the field equations are nonlinear in this 
case from the start. There are three possible terms

$$
R^{2}, R_{i k} R^{i k}, \frac{\varepsilon_{i k m n}}{\sqrt{-g}} R^{i k s t} R_{s t}^{m n}
$$

which can appear in next order in $\%$.

The term $R^{i k m n} R_{i k m n}$ can be omitted because of the identity

$$
\frac{\delta}{\delta g^{a b}}\left(R^{2}-4 R_{i k} R^{i k}+R^{i k m n} R_{i k m n}\right)=0 .
$$

A calculation of these corrections by BIRULA [7] shows that actually all three of these terms will be present. We shall consider only one of them and take as the starting point of our theory the action integral

$$
W=\int d^{4} x\left[\left(R+\frac{a^{2}}{6} R^{2}\right) \cdot \sqrt{-g}+2 x \Lambda(x)\right]
$$

where $a$ is a constant of the dimension of a length.

The field equations stemming from this Lagrangian are of fourth order and to solve them we shall resort to the weak field approximation, which will be done in Chapter 3. In Chapter 2 the work done before on $R^{2}$ terms will be discussed and in Chapter 4 we shall try to obtain experimental limits on the constant $a$ appearing in [6]. In the Appendix we prove that a theory containing only an $R^{2}$ and no $R$ term is not analytic in the coupling constant and does not have the correct Newtonian limit.

\section{Discussion of previous work}

Lagrangians of the form (6) have been investigated by EDDINGToN [1], Lanczos [2], [3] and several other workers [4], [5]. The basis of their work is that the experimental tests of General Relativity all follow from the Schwarzschild line element. Thus any theory that predicts this form of the metric in the vicinity of a mass is in agreement with experiment.

According to EDdington the Schwarzschild metric is solution of the field equations

$$
R_{i k}=R=0
$$

derivable from an action integral

as well as from

$$
W_{1}=\int d^{4} x R \cdot \sqrt{-g}
$$

$$
W_{2}=\int d^{4} x R^{2} \sqrt{-g},
$$

which is straightforward since

$$
\delta W_{2}=\int d^{4} x R(R \delta \sqrt{-g}+2 \sqrt{-g} \delta R)
$$

and thus $\delta W_{2}=0$ if $R=0$. 
It is our contention that EDINGTON's proof is not valid since one does not really want a solution of the free field equations ( 1 a) but of equations with a non-vanishing energy-momentum tensor. Since the exterior solution for any spherically symmetric mass distribution is the Schwarzschild metric in General Relativity, one usually leaves out the less interesting interior part of the solution and considers the Schwarzschild metric to be valid down to $r=0$. In the 4 th order theory containing $R^{2}$ terms there is a larger manifold of spherically symmetric vacuum solutions that are asymptotically flat. (This is in contrast to the theory containing only an $R^{2}$ term and no $R$ terms, see BuchdaHL [4]). The Schwarzschild metric is not the one that can be joined to an interior solution corresponding to a positive definite mass distribution.

To study this problem closer we shall investigate the field equations following from an action integral

$$
\bar{W}=\int d^{4} x\left(\sqrt{-g} R^{2}+\bar{x} \cdot \Lambda(x)\right)
$$

in the weak field approxmation. Following THIRRING's notation [6] we put

$$
g_{\mu \nu}=\eta_{\mu \nu}-2 \bar{f} \psi_{\mu \nu}
$$

where $\bar{f}=\sqrt{\bar{x}}$. Then the gravitational part $W_{2}$ of the action becomes ${ }^{1}$

$$
W_{2}=\int d^{4} x\left(\psi^{i k}{ }_{i k}-\psi_{i, k}^{i k}\right)^{2}(-2 \bar{f})^{2}
$$

and the field equations stemming from (7a) are easily obtained to be

$$
2\left(\psi^{i k}{ }_{, i k l m}-\psi_{l, k l m}^{l k}-\eta_{l m} \psi^{i k}{ }_{, i k n}^{n}+\eta_{l m} \psi_{i, k n}^{i k n}\right)=\bar{f} T_{l m} .
$$

If we impose the de Donder coordinate condition (Harmonic coordinates)

these equations simplify to

$$
\psi^{i k},{ }_{k}=\frac{1}{2} \psi_{k}^{k i}
$$

$$
-\psi_{i, k l m}^{i, k}+\eta_{l m} \psi_{i, k n}^{i, k n}=\bar{f} T_{l m} .
$$

For a mass point at rest at the origin the stress-energy tensor reads

$$
T_{i k c}=m \delta(\mathbf{x})\left(\begin{array}{llll}
1 & 0 & 0 & 0 \\
0 & 0 & 0 & 0 \\
0 & 0 & 0 & 0 \\
0 & 0 & 0 & 0
\end{array}\right) .
$$

Inserting this into the contracted equation (12), i.e.

$$
3 \psi_{i, k l}^{i, k}=\bar{f} T_{i}^{i}
$$

we obtain $\psi_{i, k}^{i, k} \sim \frac{1}{|\mathbf{x}|}$ and thus $\psi_{a, b 12}^{a} \neq 0$ while from (12) we get $\psi_{a, b 12}^{a b}=0$. Therefore the system (12) has no solution at all for a mass

${ }^{1} \eta_{\mu \nu}$ is the flat space-time metric $(1,-1,-1,-1)$. In all weak field calculations in this paper indices are raised and lowered by $\eta_{\mu \nu}$. A comma denotes ordinary differentiation. 
point at rest at the origin. This is of course due to the fact that the ten equations (12) contain only one variable, i.e. $\psi_{i}^{i}$. For the Schwarzschild metric $R \sim \psi_{a, b}^{a}, b \delta(\mathbf{x})$, and introducing this into (12) we see that it is a solution for $r \neq 0$. But at $r=0$ there is a $\delta^{\|}(\mathrm{x})$ singularity, i.e. the mass distribution is not positive definite, which is unacceptable. The requirements the energy-momentum tensor has to satisfy in order that a solution of (12) exists are not only $T_{i k}{ }^{k}=0$, as usual, but also

$$
\eta_{i k} \square T-T,_{i k}=3 \square T_{i k} .
$$

This is not fulfilled for an extended mass distribution of the form

$$
T_{i k}=\varrho(\mathbf{x})\left(\begin{array}{llll}
1 & 0 & 0 & 0 \\
0 & 0 & 0 & 0 \\
0 & 0 & 0 & 0 \\
0 & 0 & 0 & 0
\end{array}\right)
$$

and $\varrho(\mathbf{x})=0$ for $\mathbf{x} \geqq b$. Taking $i=k=0$ in (14) we obtain

$$
\Delta T_{00}=3 \Delta T_{00}
$$

and thus $\Delta \varrho(\mathbf{x})=0$. This implies $\varrho(\mathbf{x})=0$ as can be seen from Green's theorem

$$
\int d^{3} x u(\mathbf{x}) \Delta v(\mathbf{x})+\int(\nabla u) \cdot(\nabla v) d^{3} x=\oint u \nabla v \cdot d \boldsymbol{\sigma} .
$$

Putting $u=v=\varrho$ and integrating over a sphere of radius $b$ we obtain

$$
\int d^{3} x \varrho(\mathrm{x}) \Delta \varrho(\mathrm{x})+\int(\nabla \varrho(\mathrm{x}))^{2} d^{3} x=0 .
$$

Thus $\nabla \varrho(x)=0$ and therefore $\varrho(x)=0$. Therefore there exists no solution of (12) in the weak field approximation for an energy-momentum tensor (15). We shall study the full, nonlinear field equations stemming from the Lagrangian (7a) in the Appendix. The result is that even an infinitesimal energy-momentum tensor $T_{i k}$ leads in the $R^{2}$ theory to a strong gravitational field and to a space-time that is not asymptotically flat. Thus BuchDaHL's theorem [4] that the Schwarzschild metric is the only spherically symmetric asymptotically flat solution of the equations

$$
R R_{i k}+R_{|i| k}-g_{i k}\left(\square R+\frac{1}{4} R^{2}\right)=-\bar{\chi} T_{i k_{k}}
$$

stemming from (7a) is correct but irrelevant. The exterior solution that corresponds to a nonvanishing localized $T_{i k}$ in (16) will not be the Schwarzschild metric but a strongly curved space that is not asymptotically flat.

In parentheses we remark that the condition (15) posing a restriction on the energy-momentum tensor is present only in the linear approximation and not in the tull theory. Omitting quadratic terms in (16) leads to

$$
R_{|i| k}-g_{i k} \square R=-\bar{x} \cdot T_{i k}
$$

and here only one variable, i.e. $R$, is left, so that the equations will not have any solutions in general. 
The situation is however entirely different if we consider the field equations stemming from (6). There the linear approximation does possess solutions which are asymptotically flat. The Schwarzschild metric is of course among these solutions, but it is not the one that can be matched to an interior solution that corresponds to a meaningful energy-momentum tensor.

\section{Derivation and solution of the field equations}

In this section we will derive the field equations that stem from the variation of $g_{i k}$ in the action integral (6) and solve them for a point mass at rest at the origin. Since the exact equations are too complicated to be solved we shall immediately resort to the weak field approximation. (The exact equations will be discussed in the Appendix.)

The weak field approximation for the standard field equations (1) is in harmonic coordinates (see e.g. [5])

where $f=\sqrt{x}$.

$$
\psi_{i k, l}^{l}-\frac{1}{2} \eta_{i k} \psi_{l, m}^{l} m=f T_{i k}
$$

This expression has to be augumented by the term stemming from the $R^{2}$ part of (6), i.e. by the left-hand side of (12) multiplied by $-a^{2} / 3$. Thus the field equations read finally

$$
\psi_{i k, l}^{l}-\frac{1}{2} \eta_{i k} \psi_{l, m}^{l m}-\frac{a^{2}}{3}\left(\eta_{i k} \psi_{l, m n}^{l m n}-\psi_{l, m i k}^{l m}\right)=f T_{i k}
$$

while the contracted field equations are

$$
-a^{2} \psi_{l, m n}^{l m n}-\psi_{l, m}^{l m}=f T_{l}^{l} .
$$

Inserting the energy-momentum tensor (13) and taking into account the static character of the metric (18) becomes

$$
\Delta\left(-a^{2} \Delta+1\right) \psi_{l}^{l}=f m \delta(\mathbf{x}) .
$$

Specializing to a centrally symmetric field and introducing polar coordinates we get

$$
\left[\frac{\partial^{2}}{\partial r^{2}}+\frac{2}{r} \cdot \frac{\partial}{\partial r}-a^{2}\left(\frac{\partial^{4}}{\partial r^{4}}+\frac{4}{r} \cdot \frac{\partial^{3}}{\partial r^{3}}\right)\right] \psi_{i}^{i}=f m \delta(\mathbf{x}) .
$$

The general solution of the homogeneous part of (20) is

$$
\begin{aligned}
\psi_{i}^{i} & =c_{1}+\frac{c_{2}}{r}+\frac{c_{3}}{r} \sin ^{2}\left(\frac{r}{2|a|}\right)+\frac{c_{4}}{r} \sin \left(\frac{r}{|a|}\right) \quad a^{2}<0 \\
\psi_{i}^{i} & =c_{1}+\frac{c_{2}}{r}+\frac{c_{3}}{r} e^{-r / a}+\frac{c_{4}}{r} e^{r / a} \quad a^{2}>0 .
\end{aligned}
$$

It contains four constants of integration which can be determined from the boundary conditions at infinity (requiring $c_{1}=0$, and $c_{4}=0$ for $a^{2}>0$ ) and the conditions at the origin. 
These two requirements leave us with the following solutions for $\psi_{i}^{i}$

$$
\begin{aligned}
\psi_{i}^{i} & =-\frac{f m}{4 \pi r}+\frac{f m}{4 \pi r} e^{-r / a} \quad a^{2}>0 \\
\psi_{i}^{i} & =-\frac{f m}{2 \pi r} \sin ^{2}\left(\frac{r}{2|a|}\right)+\frac{c_{4}}{r} \sin \left(\frac{r}{|a|}\right) \quad a^{2}<0 .
\end{aligned}
$$

Thus the solution is not unique and strongly oscillating for $a^{2}<0$. Therefore we shall restrict ourselves in what follows to $a^{2}>0$.

From the above we see that without due regard to the nature of the singularity at the origin two of the four constants of integration in (21) remain undetermined. This would happen in the usual philosophy of deriving the Schwarzschild metric from $R_{i k}=0$ and requiring only boundary conditions ar infinity. Having derived $\psi_{i}^{i}$ we return to the system (17). Inserting (22) one can easily solve these field equations. The result for the line elements is

$$
\begin{aligned}
& d s^{2}=\left[1-\frac{M}{r}-\frac{M}{3 r} e^{r / a}\right] d t^{2}- \\
&- {\left[1+\frac{M}{r}+\frac{4}{3} \frac{M a^{2}}{r^{3}}-e^{-r / a}\left(\frac{M}{r}+\frac{4}{3} \frac{a M}{r^{2}}+\frac{4}{3} \frac{a^{2} M}{r^{3}}\right)\right] d r^{2}-} \\
&- r^{2}\left[1+\frac{M}{r}-\frac{2}{3} \frac{M a^{2}}{r^{3}}-e^{-r / a}\left(\frac{M}{3 r}+\frac{2}{3} \frac{a M}{r^{2}}-\frac{2}{3} \frac{M a^{2}}{r^{3}}\right)\right] d \Omega^{2} \\
& d \Omega^{2}=d \theta^{2}+\sin ^{2} \theta d \varphi^{2}
\end{aligned}
$$

where $M$ is the Schwarzschild radius of the mass $m$.

At this point we are able to show that the Schwarzschild solution is not the exterior solution for an energy-momentum tensor of the form (15) with positive definite but otherwise arbitrary $\varrho(x)$. From (22) we obtain in this case

$$
\psi_{i}^{i}(\mathbf{x})=\frac{f}{4 \pi} \int d^{3} x^{\prime} \varrho\left(\mathbf{x}^{\prime}\right)\left(-\frac{1-e^{-\left|\mathbf{x}-\mathbf{x}^{\prime}\right| / a}}{\left|\mathbf{x}-\mathbf{x}^{\prime}\right|}\right) .
$$

This is an immediate consequence of the fact that (22) is - except for the constant factor $m$ - the Green's function of (18). (24) deviates from the corresponding expression in the Schwarzschild line element by

$$
\chi(\mathbf{x})=\frac{f}{4 \pi} \int d^{3} x^{\prime} \varrho\left(\mathbf{x}^{\prime}\right) \frac{e^{-\left|\mathbf{x}-\mathbf{x}^{\prime}\right| / a}}{\left|\mathbf{x}-\mathbf{x}^{\prime}\right|} .
$$

For distances large compared to the spatial extension of the source this becomes

$$
\chi(x) \cong e^{-r / a} \cdot \frac{f}{4 \pi} \int e^{r \cos \vartheta} \varrho\left(\mathbf{x}^{\prime}\right) d^{3} x^{\prime}
$$

where $\vartheta=\Varangle\left(\mathbf{x}, \mathbf{x}^{\prime}\right)$. Since the integrand is positive definite $\chi \neq 0$ and our statement follows.

The pressure terms in $T_{i k}$ which are necessary for the hydrostatic equilibrium of a mass distribution in higher order in $\varkappa$ are of order $v^{2} / c^{2}$ 
compared to the contribution of $T_{00}$ and are therefore neglected in the linear approximation.

In General Relativity the solution of the field equations for a mass point is also the exterior solution for an arbitrary spherically symmetric mass distribution. This is not true here however. Nevertheless we can integrate the field equations for an arbitrary mass distribution of form (15). This is possible because the equations (17) are linear and are easily solved by a Green's functions method. The Green's functions are implicitly given by (23) (put $g_{i k}=\eta_{i k}+M \psi_{i k}^{(0)}$ in (23)). The solution of the field equations for an extended mass distribution is therefore

$$
\psi_{i k}(\mathbf{x})=f \int \psi_{i k}^{(0)}\left(\mathbf{x}-\mathbf{x}^{\prime}\right) \varrho\left(\mathbf{x}^{\prime}\right) d^{3} x^{\prime} .
$$

For a sphere of radius $b$ with homogeneous mass distribution $\varrho=\varrho_{0}$ $\varrho=\varrho_{0}<b \varrho=0 r>b$ the result is

where

$$
g_{00}=1-\frac{M}{p}-\frac{M}{p} e^{-r / a} n(b / a) \quad r>b
$$

$$
n(x)=x^{-2}\left(\cos h x-x^{-1} \sin h x\right) .
$$

The other $g_{i k}$ are rather complicated functions of $r$, which we shall not write out explicitly. They have the same dependence on $r$ as in (23), only the numerical values of the coefficients differ.

\section{Experimental tests of the theory}

In this section we shall discuss experimental tests of the theory and its restrictions on the constant $a$. Due to the additional term in $g_{00}$ there are deviations from the Newtonian approximation which we shall discuss before entering on the three classical tests of General Relativity.

\subsection{The Newtonian approximation}

In the non-relativistic limit only the $g_{00}$ term of the line element is of importance. From (24) we obtain the gravitational potential of a homogeneous sphere to be

$$
U=-\frac{\gamma m}{r}-\frac{\gamma m}{r} e^{-r / a} n(b / a)
$$

This differs by the second term from the standard expression. Under which conditions is this term of importance? (In the discussion below $b$ is the radius of the mass producing the gravitational field in each case; e.g. for laboratory experiments $b \sim 10 \mathrm{~cm}$, for satellite experiments $b=6370 \mathrm{~km}$.)

For distance $r \gg a$ the exponential term is obviously to be neglected while for $r-b \ll a$ it leads but to a renormalization of the gravitational constant. The observable effects are the same as in the Newtonian theory if $x$ is replaced by $\frac{3}{4} x$. Thus any experiment gives an upper and a lower bound for $a$. If $a \leqq 1 \mathrm{~cm}$, the effects can not be detected by 
present laboratory experiments. On the other hand $a \geqq 1 \mathrm{~km}$ means that the laboratory experiment will give a value $x^{\prime}=\frac{3}{4} x$ for the gravitational constant. Does this have any observable effects? Since our knowledge of the masses of the planets and stars comes only from a measurement of their gravitational fields and not from direct measurements of their inertia, nothing on an astronomical scale changes (except for star models) if we replace $x$ by $\varkappa^{\prime}$ and all masses by $\frac{3}{4} m$. Thus if $a$ has a value of say $10 \mathrm{~km}$, the real mass of the earth would not be $6 \times 10^{27} g$ but $8 \times 10^{27} g$.

Astronomical measurements set in only at larger values of $a$. If the time of revolution of a satellite around the earth can be measured to an accuracy of $10^{-6}$, then Kepler's 3rd law excludes $50 \mathrm{~km}<a<1500 \mathrm{~km}$. The same law applied to the moons and planets of the solar system excludes $1000 \mathrm{~km}<a<10^{10} \mathrm{~km}$. This latter limit is already in conflict with the validity of the weak field approximation for the $d r^{2}$ term. For the metric to be approximately flat $a^{2}$ has to be smaller than $\frac{b^{3}}{M}$ and with $M=\frac{x b^{3} \varrho}{3}$ we obtain $a^{2} \ll \frac{1}{\varkappa \varrho}$. For an average density $\varrho \sim 1 \mathrm{~g} / \mathrm{cm}^{3}$ we get $a \cdot \ll 10^{9} \mathrm{~km}$. Thus the weak field approximation breaks down for $a \gtrsim 10^{7} \mathrm{~km}$. For still larger values of $a$ the $R^{2}$ term will be the prominent one in the Lagrangian. We shall see in the appendix that in this case the metric is strongly curved even for an arbitrarily small mass distribution. This is in obvious disagreement with experiment and therefore values of $a>10^{7} \mathrm{~km}$ have to be excluded. Thus the Newtonian approximation excludes $1 \mathrm{~cm}<a<1 \mathrm{~km}$ and $50 \mathrm{~km}<a<\propto$. A value of a smaller than $1 \mathrm{~cm}$ leads to no effects that can be tested by present laboratory experiments. $1 \mathrm{~km}<a<50 \mathrm{~km}$ leads to a renormalization of the gravitational constant and thus to the prediction that the mass of the earth is $8 \times 10^{27} \mathrm{~g}$ instead of $6 \times 10^{27} \mathrm{~g}$. This is already in contradiction with geophysical evidence. Therefore, experimentally we can exclude $a \gtrsim 1 \mathrm{~cm}$.

From the experience with vacuum polarization one can estimate $a$ to be about $10^{-13} \mathrm{~cm}$.

\subsection{Relativistic effects}

The three tests of General Relativity give us no new restrictions on $a$. The red shift, being a consequence of the principle of equivalence tests nothing but the Newtonian approximation.

The deflection of light is changed from $\delta=\frac{2 M}{R}$ to $\delta=\frac{M}{R}\left(1-0.3 \frac{a}{R}\right)$ for $a / R \geqq 5$. This excludes $a>2 \times 10^{6} \mathrm{~km}$.

Our approximation scheme does not permit us to calculate the perihelion advance, since this effect is beyond the linear approximation. 


\section{Some remarks on elementary particles}

Since the effects discussed in this paper stem from vacuum polarization, which is a quantum phenomenon, their most important application should be in the field of elementary particle physics. The calculations of BIRULA [7] indicate for $a$ the value (we put $\hbar=1, m$ is the electron mass)

$$
a=\frac{e^{2}}{m} \cong 10^{-13} \mathrm{~cm} \text {. }
$$

Let us consider an electron, possessing a Schwarzschild-radius $M=\varkappa \cdot m \cong$ $\cong 10^{-56} \mathrm{~cm}$. This means that the gravitational phenomena become important only at that small $a$ radius, this being therefore the minimum size for a bare electron (bare in this connection refers to the electromagnetic interactions).

If we consider, however, (23) to be the line element in the neighborhood of the electron, the singularity with largest radius is due to the $-\frac{4}{3} \frac{a M}{r^{2}} e^{-r / a}$ term in $d r^{2}$. This term becomes unity for

$$
r \cong \sqrt{M \cdot a}=\sqrt{x \cdot m \cdot e^{2} / m} \cong 10^{-34} \mathrm{~cm} .
$$

Thus the quantum phenomena push out the effective radius of the electron from $10^{-56} \mathrm{~cm}$ to $10^{-34} \mathrm{~cm}$. Since our calculations was done in the weak field approximation we can not really tell whether a singularity occurs at that radius or not. What we can say is just that gravitational phenomena should become important at a radius as large as the one given above.

We should like to thank Prof. W. ThIRRINg for his constant interest in this work and Prof. A. Trautman and Prof. H. Buchdahl for valuable comments.

\section{Appendix \\ The nonlinear field equations}

In this section we shall give a short discussion of the full field equations (16) with special regard to the role of the energy-momentum tensor, which turns out to be crucial. Our starting point is (16)

$$
R R_{i k}+R_{|i| k}-g_{i k}\left(\square R+\frac{1}{4} R^{2}\right)=-\bar{\varkappa} T_{i k} .
$$

Upon contraction we get

$$
3 \square R=\bar{*} T
$$

and thus (A.1) can be rewritten as

$$
R\left(R_{i k}-\frac{1}{4} g_{i k} R\right)=-\bar{\varkappa} T_{i k}-R_{|i| k}+\frac{\bar{x}}{3} g_{i k} T .
$$

We now introduce a quantity $D$ by

$$
D=T
$$


and obtain

$$
R_{i k}-\frac{1}{4} g_{i k} R=-\frac{1}{D}\left(3 T_{i k}+D_{|i| k}-g_{i k} T\right) .
$$

The most remarkable feature about (A.5) is that the graviational constant has dropped out. To rewrite the field equations in a form completely analogous to Ernstern's equations we use again (A.2) and (A.4) to obtain

$$
R_{i k}-\frac{1}{2} g_{i k} R=\frac{1}{D}\left(-3 T_{i k}-D_{|i| k}+g_{i k} T\right)-\frac{1}{4} g_{i k} \frac{\tilde{x}}{3} D \text {. }
$$

Here the dependence on $\bar{x}$ has reappeared. Putting the right hand side equal to $-x \Pi_{i k}$ we obtain

$$
\begin{gathered}
R_{i k}-\frac{1}{2} g_{i k} R=-x \Pi_{i k} \\
\Pi_{i k}=\frac{1}{x D}\left(3 T_{i k}+D_{|i| k}-g_{i k} T\right)+\frac{1}{12} g_{i k} D \frac{\bar{x}}{\varkappa} .
\end{gathered}
$$

$\Pi_{i k}$ is thus the energy momentum tensor that appears in the corresponding equations of General Relativity. We therefore can treat the field equations (A.1) in the framework of the usual Einstein theory. We only have to replace $T_{i k}$ by $\Pi_{i k}$. Of course the progress made is only formal, because we have to know the metric before we can solve the equation (A.4) for D. A formal solution of (A.4) is

$$
D(x)=\int G\left(x, x^{\prime}\right) T\left(x^{\prime}\right) d^{4} x^{\prime}
$$

where $G$ is the Green's function of the $\square$-operator. $D$ is a nonlocal quantity different from zero even outside the mass distribution, where we have for $\Pi_{i k}$

$$
\Pi_{i k}=\frac{D_{|i| k}}{\varkappa D}+\frac{1}{12} g_{i k} D \frac{\bar{x}}{\varkappa} .
$$

Now we have to make an important point. For a localized not-too-dense mass distribution the metric has to be flat, to a very good approximation, in the interior and the exterior of the body. This just means that Euclidean geometry is an excellent approximation in the interior and exterior of the earth e.g. This is an observational fact not to be confused with the weak field approximation used before to treat (1) or (16).

We shall show that the full nonlinear equations (A.1) have no solutions that are flat in the above sense. Let us assume such a solution would exist. Then the Green's function in (A.9) can be replaced in an excellent approximation by the one of the flat $\square$-operator, yielding $D \approx \frac{m}{4 \pi r}$, where $m$ is the mass of the body. (This is valid for $r>\varrho$ where $\varrho$ is the radius of the spherically symmetric body.) Therefore $D_{|i| k} \approx \frac{m}{4 \pi} \frac{x_{i} x_{k}}{r^{5}} i, k=1,2,3$ and zero otherwise. Inserting this into (A.10) we obtain in the exterior of the body

$$
\Pi_{i k}=\frac{x_{i} x_{k}}{r^{4} \varkappa_{k}}\left(1-\delta_{i}^{0}\right)\left(1-\delta_{k}^{0}\right)+\frac{1}{12} g_{i k} \frac{m}{r} \frac{\bar{x}}{\varkappa} .
$$


Thus the local energy momentum tensor $T_{i k}$ gives rise to an energymomentum distribution $\Pi_{i k}$ in (A.7) that is smeared out over all of the space. Since the space integral of (A.12) diverges, the total mass that enters into (A.7) will not be finite. Hence space-time will be strongly curved, and not asymptotically flat. Therefore our initial assumption that space-time is approximately flat in the presence of very small masses leads to a contradiction. Thus even an infinitesimal energymomentum tensor leads to a strongly curved space in this theory. Therefore the theory defined by the Lagrangian (7a) cannot be used as a realistic theory of gravitation. It is, however, an interesting example of a classical theory which possesses solutions that depend singularly on the coupling constant.

\section{References}

[1] Eddington, A. S.: Relativitätstheorie in mathematischer Behandlung. Berlin: J. Springer 1925.

[2] Lanczos, C.: Z. Phys. 73, 147 (1932).

[3] - Ann. Math. 39, 842 (1938).

[4] BUCHDAHL, H.: Nuovo cimento 128, 141 (1962).

[5] Gregory, C.: Phys. Rev. 72, 72 (1947).

[6] Thirring, W.: Ann. Phys. 16, 91 (1961).

[7] Birdla, I.: Private communication. 